\title{
Superior Infrared Nonlinear Optical Performance Achieved by Synergetic Functional Motif and Vacancy Site Modulations
}

Shao-Min Pei ${ }^{\sharp}, a, b$, Bin-Wen Liu ${ }^{\ddagger}$, , Xiao-Ming Jiang ${ }^{*}, a$, Ya-Qian Zou ${ }^{a}$,Wen-Fa Chen ${ }^{a}$, QiuNan Yan ${ }^{a}$ and Guo-Cong Guo*,a

${ }^{a}$ State Key Laboratory of Structural Chemistry, Fujian Institute of Research on the Structure of Matter, Chinese Academy of Sciences, Fuzhou, Fujian 350002

${ }^{b}$ A University of Chinese Academy of Sciences, Beijing 100190

* Guo-Cong Guo: gcguo@fjirsm.ac.cn; Xiao-Ming Jiang: xmjiang@fjirsm.ac.cn.

I Shao-Min Pei and Bin-Wen Liu contributed equally to this paper. 


\section{Computation Procedures.}

Two definitions were used to measure the degree of the distortions of the coordination polyhedral in $\mathbf{1}$ and 2. One is $\Delta H$, proposed by Lalik using Shannon's information theory. ${ }^{1}$ $\Delta H=\left(\frac{A}{V}\right) \sum_{i}\left[s_{i} \ln \left(\frac{s_{i}}{s^{\prime}}\right)\right]=A<\left(\frac{s_{i}}{s^{\prime}}\right) \ln \left(\frac{s_{i}}{s^{\prime}}\right)>$, where the summation is over the $N$ bonds in the coordination polyhedron, $>$ indicates an average over the coordinated bonds, $A$ is a constant equal to $1 / \ln 2, V$ is the formal oxidation state of the central cation, $s_{i}$ is the valence of the ith bond and $s^{\prime}$ is the average valence of the bonds in the coordination sphere. The bond valence $s_{i}$ was calculated using $s_{i}=\exp \left[\left(R_{o}-R_{i}\right) / B\right],{ }^{2}$ where $R_{\mathrm{o}}$ and $B$ are constants determined empirically for each type of bond, with the former being the notional length of a bond of unit valence and the latter a measure of the softness of the bond. These parameters were tabulated for most bond types by Brown. ${ }^{3}$ The other one is $\Delta R$ proposed by Brown., $\Delta R=-(B N) i \ln \left(\frac{s_{i}}{s}\right)=-B<$ $\ln \frac{s_{i}}{s^{\prime}}>$, where $B, N, s_{i}$, and $s^{\prime}$ have the same meanings as above. ${ }^{4}$

\section{Figures and Tables.}
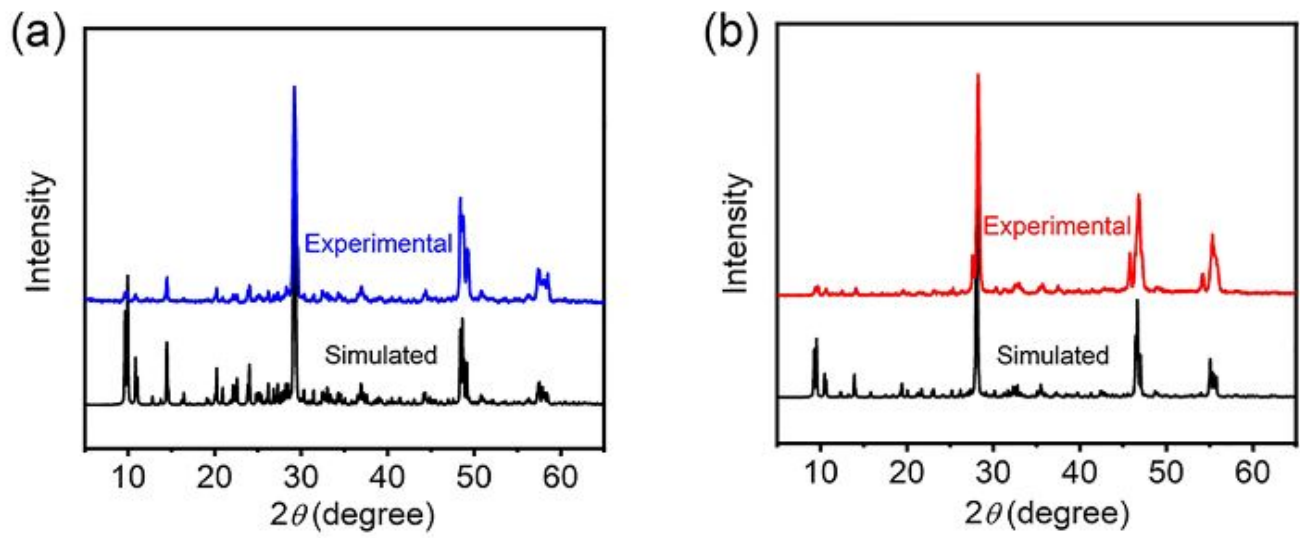

Figure S1. Experimental and simulated powder XRD patterns of 1 (a) and 2 (b). 
(a)

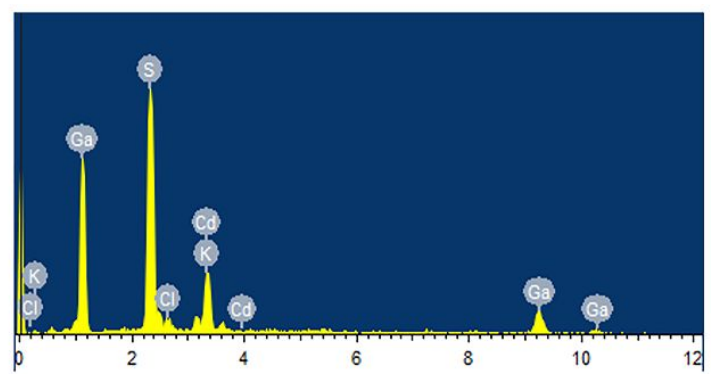

(b)

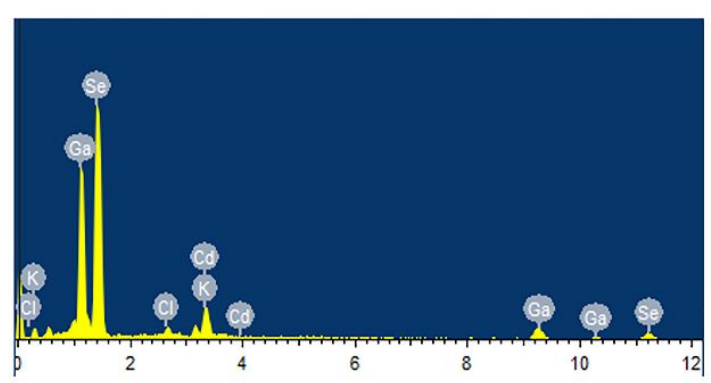

Figure S2. EDS spectra of single-crystals of 1 (a) and 2 (b).
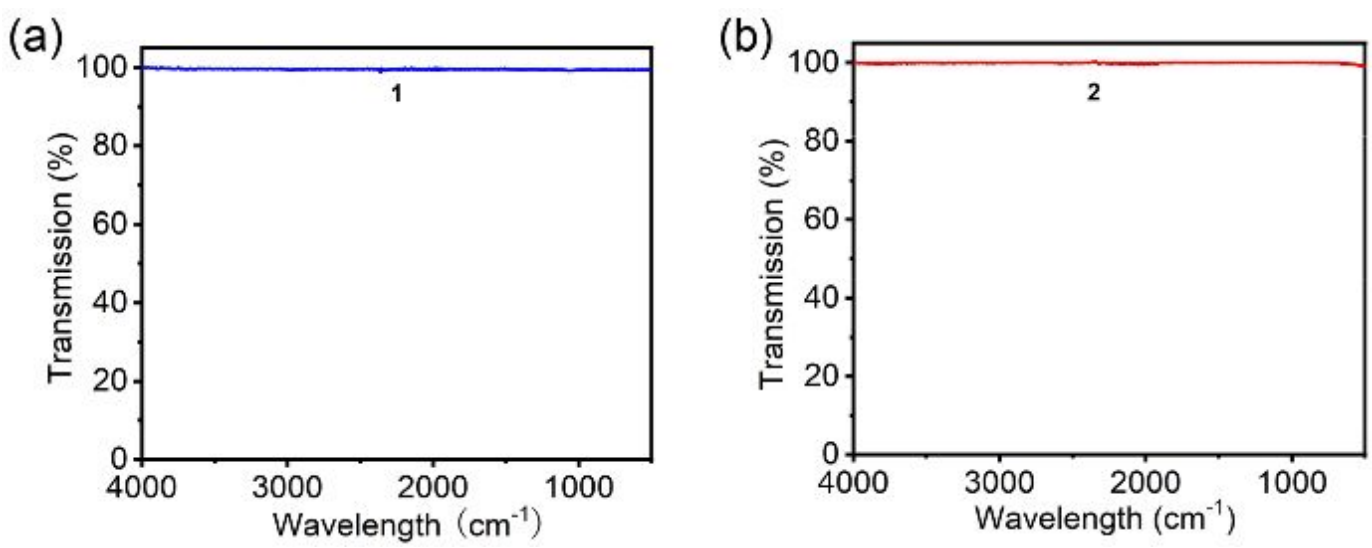

Figure S3. IR spectra of 1 (a) and 2 (b). 

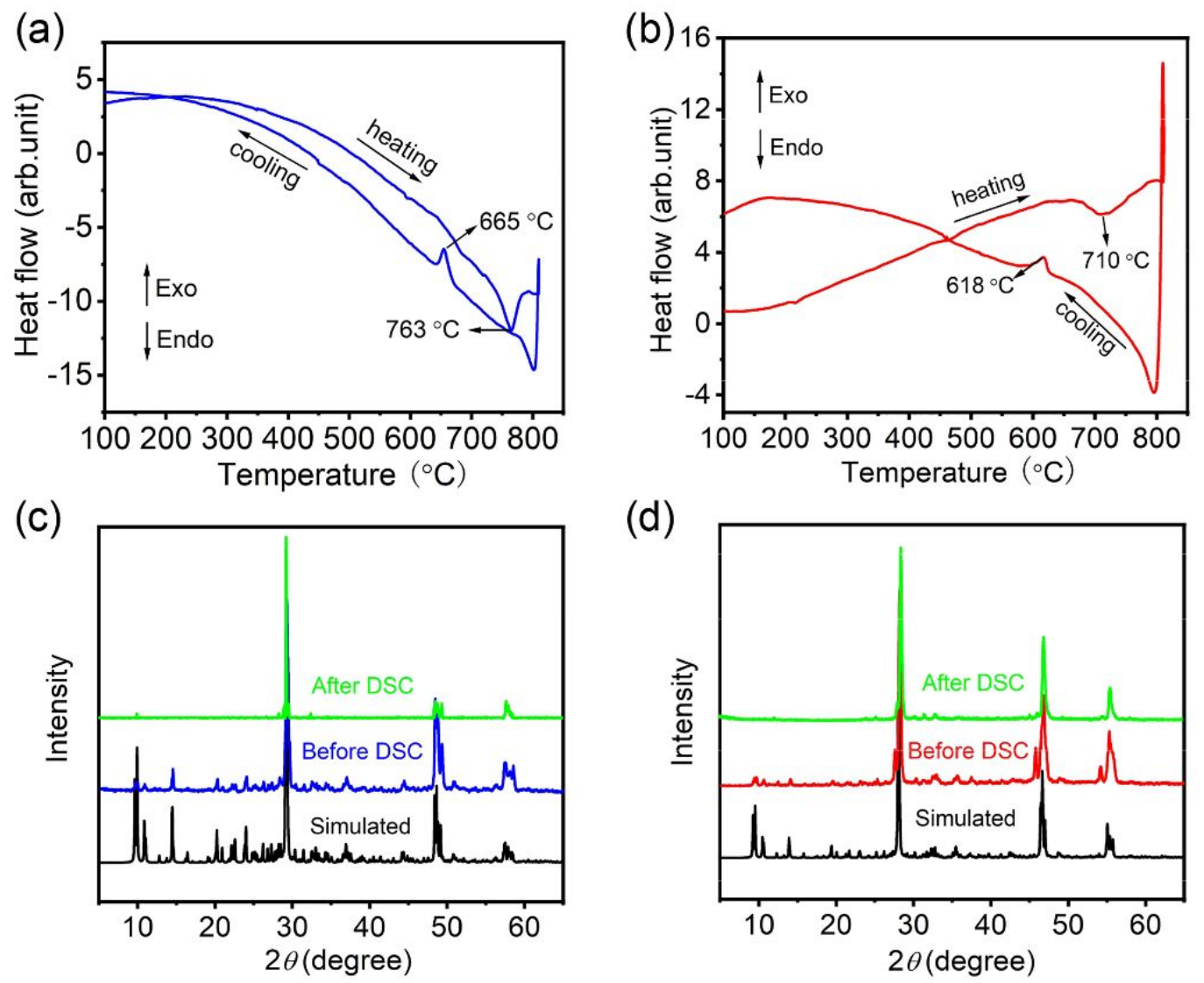

Figure S4. DSC curves of 1 (a) and 2 (b) upon heating and followed by cooling. Powder XRD patterns of the simulated, before DSC and after DSC measurements for $\mathbf{1}$ (c) and $\mathbf{2}$ (d).
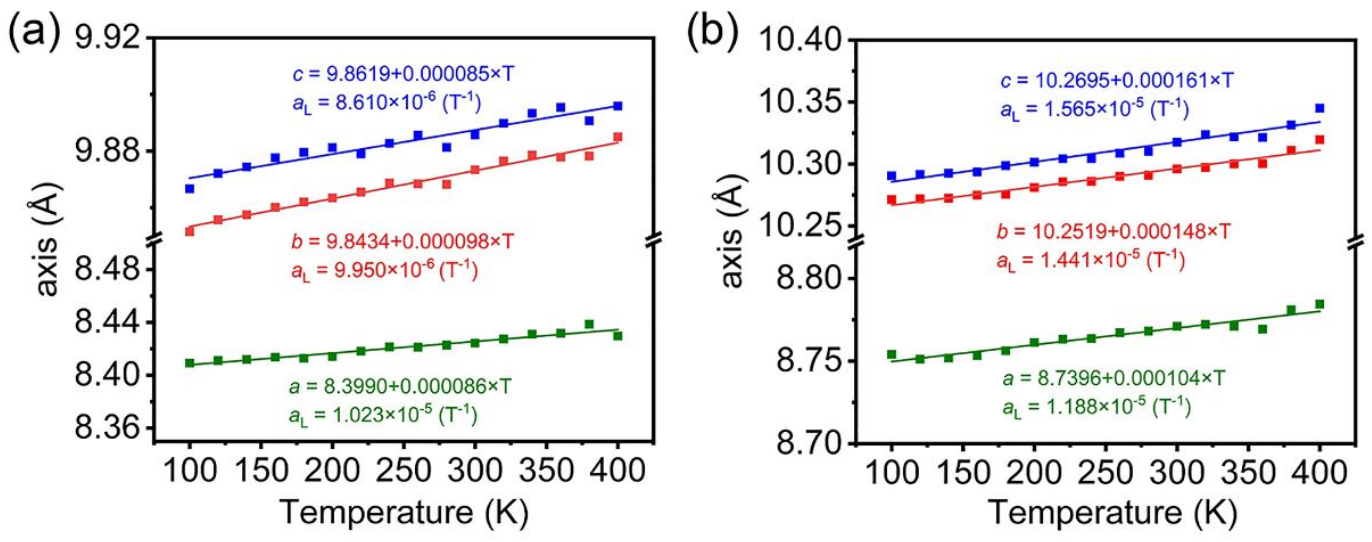

Figure S5. Temperature variation of the crystal lattice parameters ( $a, b$, and $c$ axis) of $\mathbf{1}$ (a) and 2 (b). The values of $\alpha_{\mathrm{L}}$ near the curves are thermal expansion coefficients along the corresponding axes. 
(a)

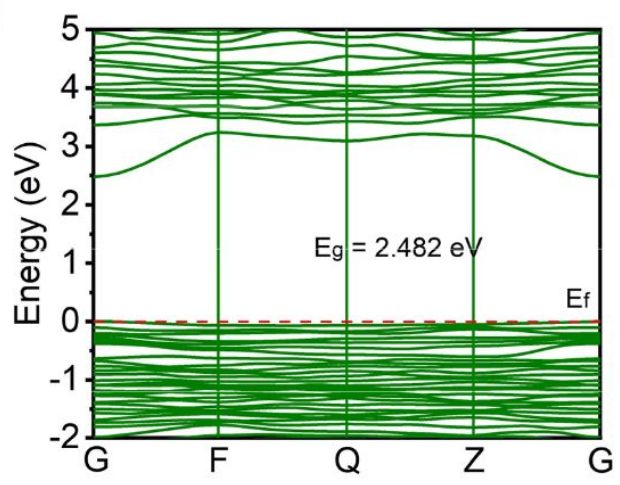

(b)

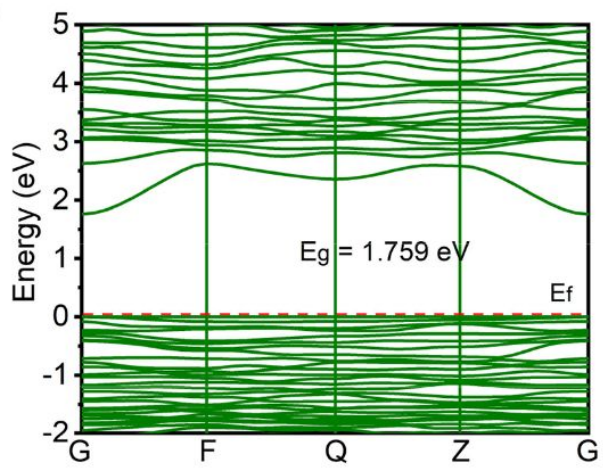

Figure S6. Electronic band structures of 1 (a) and 2 (b).

(a)

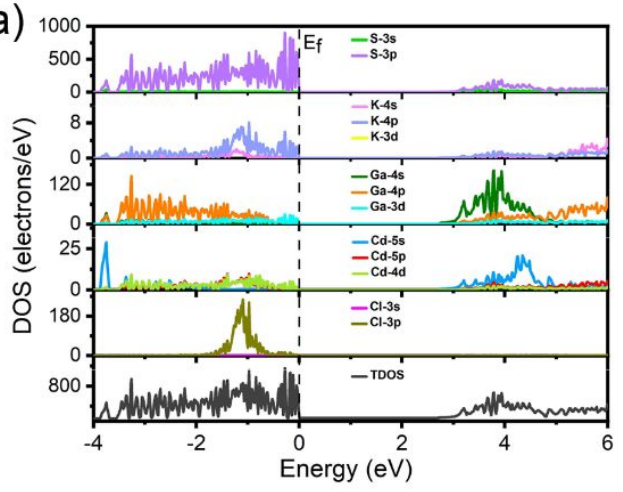

(b)

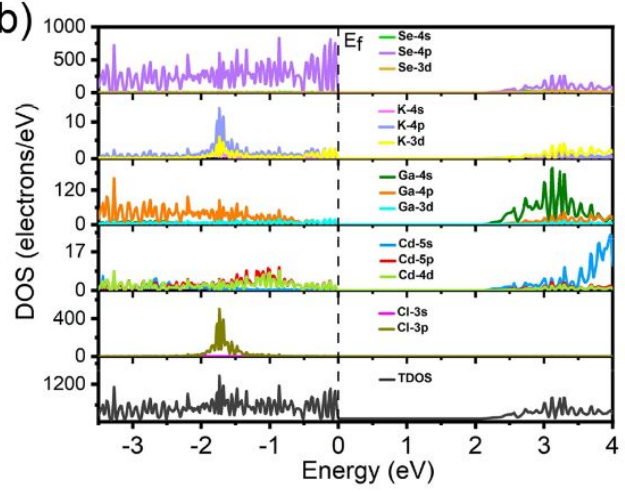

Figure S7. Density of states of 1 (a) and 2 (b).

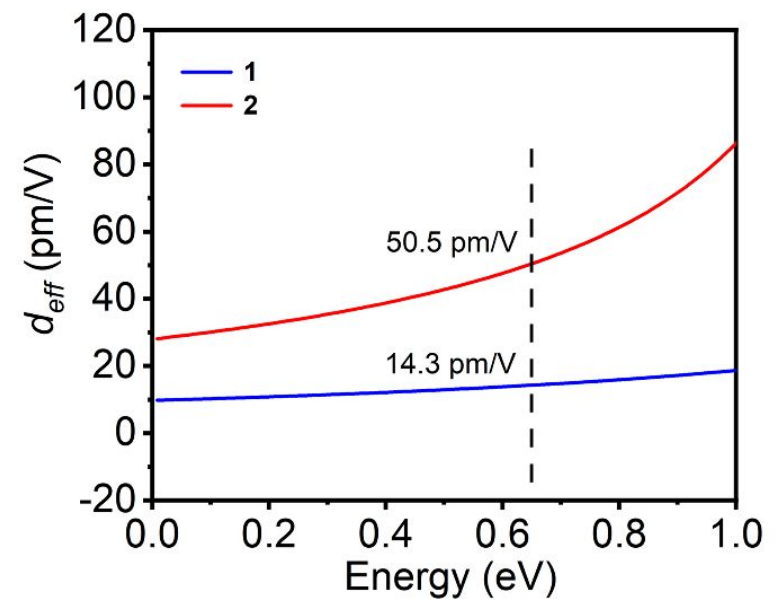

Figure S8. Calculated NLO coeffcient tensors of $\mathbf{1}$ and $\mathbf{2}$. 
(a)

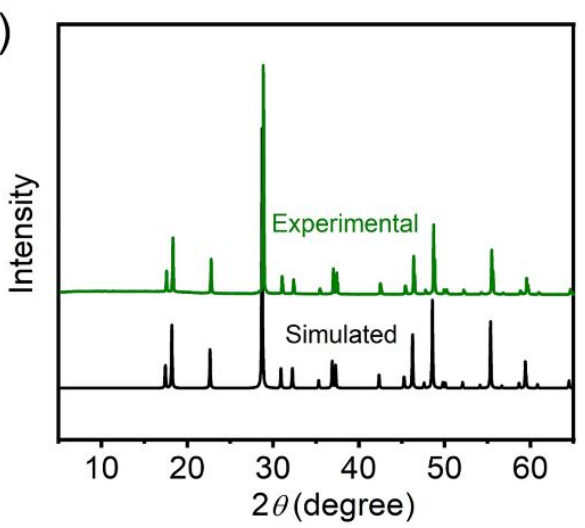

(c)

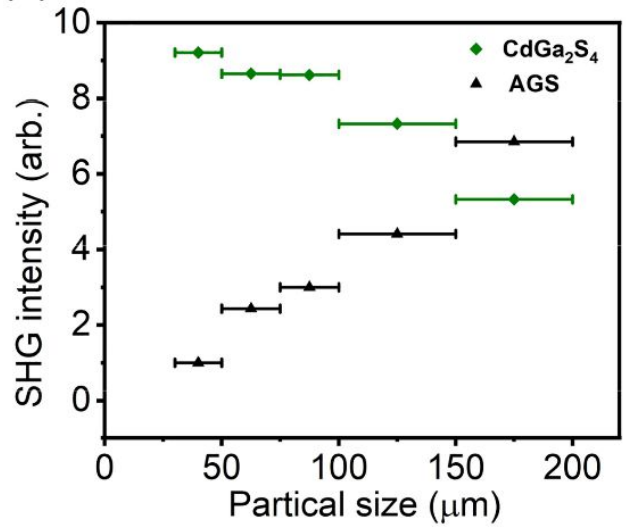

(b) 0.5

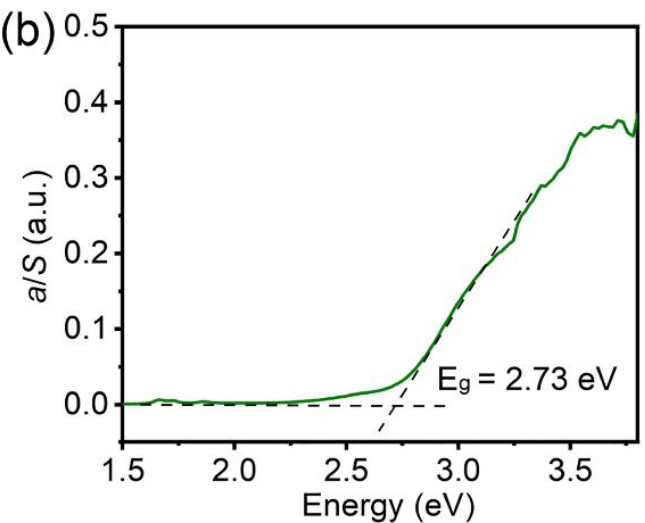

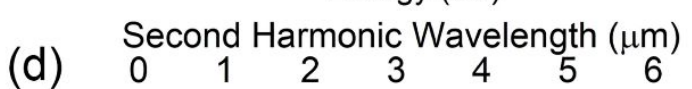

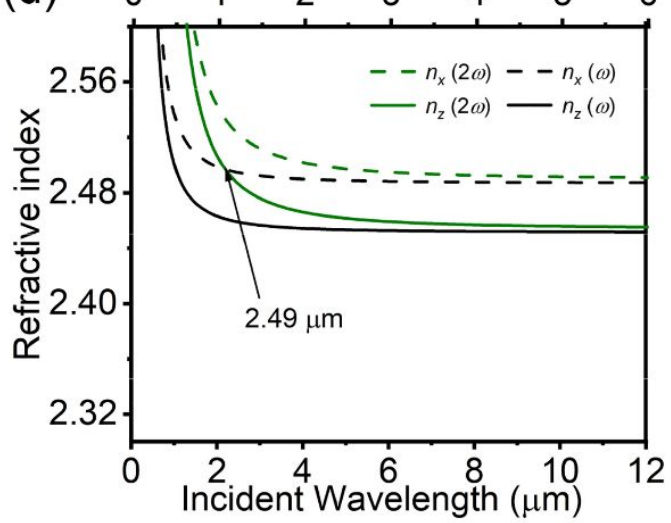

Figure S9. (a) Experimental and simulated powder XRD patterns of $\mathrm{CdGa}_{2} \mathrm{~S}_{4}$. (b) Absorption spectra of $\mathrm{CdGa}_{2} \mathrm{~S}_{4}$ from diffuse reflectance spectra. (c) Non-phase-matching behavior of $\mathrm{CdGa}_{2} \mathrm{~S}_{4}$ at the incident laser of $1910 \mathrm{~nm}$. (d) Calculated frequency-dependent refractive indexes of $\mathrm{CdGa}_{2} \mathrm{~S}_{4}$.

Table S1. Atomic coordinates, equivalent isotropic displacement parameters $\left(\AA^{2}\right)$ and bond valance sums (BVS) for $\mathbf{1}$ and $\mathbf{2}$.

1

\begin{tabular}{lllllll}
\hline Atom & $x$ & $y$ & $z$ & $U_{(\mathrm{eq})}{ }^{2}\left(\AA^{2}\right)$ & Wyckoff & BVS \\
\hline $\mathrm{K}(1)$ & $0.1357(4)$ & $0.1367(3)$ & $0.4017(3)$ & $0.0321(7)$ & $1 \mathrm{a}$ & 1.082 \\
$\mathrm{~K}(2)$ & $1.0000(4)$ & $1.0000(3)$ & $1.0000(3)$ & $0.0332(7)$ & $1 \mathrm{a}$ & 1.031 \\
$\mathrm{~K}(3)$ & $0.7884(4)$ & $0.4311(4)$ & $0.0937(3)$ & $0.0424(8)$ & $1 \mathrm{a}$ & 1.015 \\
$\mathrm{~K}(4)$ & $0.3710(4)$ & $0.3557(3)$ & $0.9282(4)$ & $0.0374(8)$ & $1 \mathrm{a}$ & 1.014 \\
$\mathrm{Cd}(1)$ & $0.28419(11)$ & $0.69883(9)$ & $0.54787(9)$ & $0.0138(2)$ & $1 \mathrm{a}$ & 2.289 \\
\hline
\end{tabular}




\begin{tabular}{|c|c|c|c|c|c|c|}
\hline $\mathrm{Ga}(1)$ & $0.98532(16)$ & $0.39646(14)$ & $0.64346(14)$ & $0.0082(3)$ & $1 \mathrm{a}$ & 2.886 \\
\hline $\mathrm{Ga}(2)$ & $0.40035(16)$ & $0.79562(14)$ & $0.85087(14)$ & $0.0088(3)$ & $1 \mathrm{a}$ & 2.922 \\
\hline $\mathrm{Ga}(3)$ & $0.82183(17)$ & $0.75157(16)$ & $0.69326(16)$ & $0.0116(3)$ & $1 \mathrm{a}$ & 2.994 \\
\hline $\mathrm{Ga}(4)$ & $0.58445(16)$ & $0.99851(14)$ & $0.43779(14)$ & $0.0082(3)$ & $1 \mathrm{a}$ & 2.868 \\
\hline $\mathrm{Ga}(5)$ & $0.73830(16)$ & $0.65312(13)$ & $0.38421(14)$ & $0.0113(3)$ & $1 \mathrm{a}$ & 2.869 \\
\hline $\mathrm{Ga}(6)$ & $0.70369(16)$ & $0.09727(13)$ & $0.73018(13)$ & $0.0079(3)$ & $1 \mathrm{a}$ & 2.861 \\
\hline $\mathrm{Ga}(7)$ & $0.42653(15)$ & $0.35498(14)$ & $0.48855(14)$ & $0.0110(3)$ & $1 \mathrm{a}$ & 2.900 \\
\hline $\mathrm{Ga}(8)$ & $0.20799(16)$ & $0.60476(12)$ & $0.22983(13)$ & $0.0088(3)$ & $1 \mathrm{a}$ & 2.881 \\
\hline $\mathrm{Ga}(9)$ & $0.50578(17)$ & $0.89452(14)$ & $0.14710(14)$ & $0.0093(3)$ & $1 \mathrm{a}$ & 2.849 \\
\hline$S(1)$ & $0.7600(4)$ & $0.2707(3)$ & $0.8064(3)$ & $0.0138(6)$ & $1 \mathrm{a}$ & 1.600 \\
\hline $\mathrm{S}(2)$ & $0.5191(4)$ & $0.5217(3)$ & $0.5610(3)$ & $0.0124(6)$ & $1 \mathrm{a}$ & 1.982 \\
\hline $\mathrm{S}(3)$ & $0.9576(4)$ & $0.4935(3)$ & $0.3956(3)$ & $0.0109(6)$ & $1 \mathrm{a}$ & 1.819 \\
\hline$S(4)$ & $0.4595(4)$ & $0.9950(3)$ & $0.9049(3)$ & $0.0097(6)$ & $1 \mathrm{a}$ & 1.826 \\
\hline$S(5)$ & $0.5389(4)$ & $0.0886(3)$ & $0.2074(3)$ & $0.0156(7)$ & $1 \mathrm{a}$ & 1.575 \\
\hline$S(6)$ & $0.3501(4)$ & $0.9041(3)$ & $0.6155(3)$ & $0.0119(6)$ & $1 \mathrm{a}$ & 1.979 \\
\hline$S(7)$ & $0.6521(4)$ & $0.1938(3)$ & $0.4902(3)$ & $0.0096(6)$ & $1 \mathrm{a}$ & 1.813 \\
\hline $\mathrm{S}(8)$ & $0.5981(4)$ & $0.6312(3)$ & $0.8539(3)$ & $0.0171(7)$ & $1 \mathrm{a}$ & 1.720 \\
\hline $\mathrm{S}(9)$ & $0.8066(4)$ & $0.8435(3)$ & $0.4425(3)$ & $0.0109(6)$ & $1 \mathrm{a}$ & 1.946 \\
\hline $\mathrm{S}(10)$ & $0.0222(3)$ & $0.5777(3)$ & $0.7256(3)$ & $0.0111(6)$ & $1 \mathrm{a}$ & 1.978 \\
\hline$S(11)$ & $0.7188(4)$ & $0.7410(3)$ & $0.1473(3)$ & $0.0166(6)$ & $1 \mathrm{a}$ & 1.666 \\
\hline$S(12)$ & $0.2474(4)$ & $0.8046(3)$ & $0.2838(3)$ & $0.0124(6)$ & $1 \mathrm{a}$ & 1.937 \\
\hline$S(13)$ & $0.2030(4)$ & $0.2483(3)$ & $0.6676(3)$ & $0.0155(6)$ & $1 \mathrm{a}$ & 1.609 \\
\hline $\mathrm{S}(14)$ & $0.1618(4)$ & $0.6848(3)$ & $0.9997(3)$ & $0.0128(6)$ & $1 \mathrm{a}$ & 1.607 \\
\hline $\mathrm{S}(15)$ & $0.9129(4)$ & $0.9436(3)$ & $0.7208(3)$ & $0.0142(6)$ & $1 \mathrm{a}$ & 1.648 \\
\hline $\mathrm{S}(16)$ & $0.3937(4)$ & $0.4254(3)$ & $0.2547(3)$ & $0.0151(6)$ & $1 \mathrm{a}$ & 1.614 \\
\hline $\mathrm{Cl}(1)$ & $0.0527(4)$ & $0.2208(4)$ & $0.1165(4)$ & $0.0276(7)$ & $1 \mathrm{a}$ & 1.364 \\
\hline \multicolumn{7}{|c|}{2} \\
\hline Atom & $x$ & $y$ & $z$ & $U_{(\mathrm{eq})}^{2}\left(\AA^{2}\right)$ & Wyckoff & BVS \\
\hline $\mathrm{K}(1)$ & $0.8570(6)$ & $0.8645(4)$ & $0.6189(5)$ & $0.0413(13)$ & $1 \mathrm{a}$ & 1.008 \\
\hline
\end{tabular}




\begin{tabular}{|c|c|c|c|c|c|c|}
\hline $\mathrm{K}(2)$ & $0.0000(6)$ & $0.0000(4)$ & $0.0000(5)$ & $0.0400(12)$ & $1 \mathrm{a}$ & 0.929 \\
\hline $\mathrm{K}(3)$ & $0.1956(6)$ & $0.5830(5)$ & $0.9184(5)$ & $0.0478(14)$ & $1 \mathrm{a}$ & 1.023 \\
\hline $\mathrm{K}(4)$ & $0.6364(6)$ & $0.6471(5)$ & $0.0852(6)$ & $0.0454(14)$ & $1 \mathrm{a}$ & 0.983 \\
\hline $\mathrm{Cd}(1)$ & $0.70351(19)$ & $0.30570(14)$ & $0.46933(16)$ & $0.0212(4)$ & $1 \mathrm{a}$ & 2.269 \\
\hline $\mathrm{Ga}(1)$ & $0.0049(2)$ & $0.60755(19)$ & $0.3712(2)$ & $0.0144(5)$ & $1 \mathrm{a}$ & 2.984 \\
\hline $\mathrm{Ga}(2)$ & $0.5909(2)$ & $0.2097(2)$ & $0.1619(2)$ & $0.0159(5)$ & $1 \mathrm{a}$ & 3.018 \\
\hline $\mathrm{Ga}(3)$ & $0.1669(3)$ & $0.2527(2)$ & $0.3201(3)$ & $0.0170(5)$ & $1 \mathrm{a}$ & 3.075 \\
\hline $\mathrm{Ga}(4)$ & $0.4065(3)$ & $0.0074(2)$ & $0.5749(2)$ & $0.0143(5)$ & $1 \mathrm{a}$ & 2.968 \\
\hline $\mathrm{Ga}(5)$ & $0.2531(2)$ & $0.35134(19)$ & $0.6296(2)$ & $0.0159(5)$ & $1 \mathrm{a}$ & 2.960 \\
\hline $\mathrm{Ga}(6)$ & $0.2870(3)$ & $0.90765(19)$ & $0.2817(2)$ & $0.0151(5)$ & $1 \mathrm{a}$ & 2.960 \\
\hline $\mathrm{Ga}(7)$ & $0.5643(2)$ & $0.6503(2)$ & $0.5259(2)$ & $0.0162(5)$ & $1 \mathrm{a}$ & 2.976 \\
\hline $\mathrm{Ga}(8)$ & $0.7831(3)$ & $0.39816(18)$ & $0.7837(2)$ & $0.0159(5)$ & $1 \mathrm{a}$ & 2.986 \\
\hline $\mathrm{Ga}(9)$ & $0.4834(3)$ & $0.1107(2)$ & $0.8666(2)$ & $0.0149(5)$ & $1 \mathrm{a}$ & 2.956 \\
\hline $\operatorname{Se}(1)$ & $0.2333(2)$ & $0.73168(17)$ & $0.2025(2)$ & $0.0158(5)$ & $1 \mathrm{a}$ & 1.630 \\
\hline $\operatorname{Se}(2)$ & $0.4731(2)$ & $0.48349(16)$ & $0.44675(19)$ & $0.0160(5)$ & $1 \mathrm{a}$ & 2.039 \\
\hline $\operatorname{Se}(3)$ & $0.0339(2)$ & $0.51715(17)$ & $0.62053(18)$ & $0.0131(5)$ & $1 \mathrm{a}$ & 1.899 \\
\hline $\operatorname{Se}(4)$ & $0.5369(2)$ & $0.00642(17)$ & $0.10848(19)$ & $0.0132(4)$ & $1 \mathrm{a}$ & 1.911 \\
\hline $\operatorname{Se}(5)$ & $0.4468(2)$ & $0.91244(17)$ & $0.8101(2)$ & $0.0193(5)$ & $1 \mathrm{a}$ & 1.624 \\
\hline $\operatorname{Se}(6)$ & $0.6465(2)$ & $0.09926(17)$ & $0.39902(19)$ & $0.0147(4)$ & $1 \mathrm{a}$ & 2.024 \\
\hline $\operatorname{Se}(7)$ & $0.3322(2)$ & $0.80881(17)$ & $0.5266(2)$ & $0.0130(5)$ & $1 \mathrm{a}$ & 1.878 \\
\hline $\operatorname{Se}(8)$ & $0.3920(2)$ & $0.37780(18)$ & $0.1570(2)$ & $0.0191(5)$ & $1 \mathrm{a}$ & 1.770 \\
\hline $\operatorname{Se}(9)$ & $0.1768(2)$ & $0.15937(18)$ & $0.5739(2)$ & $0.0145(4)$ & $1 \mathrm{a}$ & 2.027 \\
\hline $\operatorname{Se}(10)$ & $0.9621(2)$ & $0.42821(16)$ & $0.2853(2)$ & $0.0154(4)$ & $1 \mathrm{a}$ & 1.994 \\
\hline $\operatorname{Se}(11)$ & $0.2695(3)$ & $0.26669(19)$ & $0.8706(2)$ & $0.0190(4)$ & $1 \mathrm{a}$ & 1.719 \\
\hline $\operatorname{Se}(12)$ & $0.7483(2)$ & $0.19560(17)$ & $0.72878(19)$ & $0.0165(5)$ & $1 \mathrm{a}$ & 1.995 \\
\hline $\operatorname{Se}(13)$ & $0.7887(2)$ & $0.76317(18)$ & $0.3439(2)$ & $0.0188(5)$ & $1 \mathrm{a}$ & 1.637 \\
\hline $\operatorname{Se}(14)$ & $0.8357(2)$ & $0.3187(2)$ & $0.0145(2)$ & $0.0181(4)$ & $1 \mathrm{a}$ & 1.639 \\
\hline $\operatorname{Se}(15)$ & $0.0717(2)$ & $0.06107(17)$ & $0.2886(2)$ & $0.0181(5)$ & $1 \mathrm{a}$ & 1.694 \\
\hline $\operatorname{Se}(16)$ & $0.5936(2)$ & $0.57872(17)$ & $0.76368(19)$ & $0.0187(5)$ & $1 \mathrm{a}$ & 1.673 \\
\hline
\end{tabular}




\begin{tabular}{lllllll}
$\mathrm{Cl}(1)$ & $0.9360(6)$ & $0.7843(5)$ & $0.8947(6)$ & $0.0322(11)$ & $1 \mathrm{a}$ & 1.295 \\
\hline
\end{tabular}

Table S2. Selected bond distances $(\AA)$ for $\mathbf{1}$ and $\mathbf{2}$.

\begin{tabular}{|c|c|c|c|c|c|}
\hline \multicolumn{6}{|c|}{1} \\
\hline bond & distance & bond & distance & bond & distance \\
\hline $\mathrm{Cd} 1-\mathrm{S} 10$ & $2.500(3)$ & Ga1-S13 & $2.248(3)$ & Ga2-S8 & $2.220(3)$ \\
\hline Cd1-S6 & $2.504(3)$ & Ga1-S1 & $2.251(3)$ & Ga2-S14 & $2.245(3)$ \\
\hline $\mathrm{Cd} 1-\mathrm{S} 2$ & $2.514(3)$ & Ga1-S10 & $2.297(3)$ & Ga2-S6 & $2.320(3)$ \\
\hline $\mathrm{Cd} 1-\mathrm{S} 12$ & $2.525(3)$ & Ga1-S3 & $2.348(3)$ & Ga2-S4 & $2.345(3)$ \\
\hline Ga3-S8 & $2.218(3)$ & Ga4-S5 & $2.238(3)$ & Ga5-S11 & $2.220(3)$ \\
\hline Ga3-S15 & $2.238(3)$ & Ga4-S6 & $2.273(3)$ & Ga5-S2 & $2.285(3)$ \\
\hline Ga3-S10 & $2.299(3)$ & Ga4-S9 & $2.313(3)$ & Ga5-S9 & $2.318(3)$ \\
\hline Ga3-S9 & $2.339(3)$ & Ga4-S7 & $2.326(3)$ & Ga5-S3 & $2.331(3)$ \\
\hline Ga6-S15 & $2.231(3)$ & Ga7-S16 & $2.238(3)$ & Ga8-S14 & $2.243(3)$ \\
\hline Ga6-S1 & $2.241(3)$ & Ga7-S13 & $2.239(3)$ & Ga8-S16 & $2.246(3)$ \\
\hline Ga6-S4 & $2.338(3)$ & Ga7-S2 & $2.297(3)$ & Ga8-S12 & $2.296(3)$ \\
\hline Ga6-S7 & $2.356(3)$ & Ga7-S7 & $2.368(3)$ & Ga8-S3 & $2.366(3)$ \\
\hline Ga9-S11 & $2.241(3)$ & Ga9-S5 & $2.264(3)$ & Ga9-S12 & $2.302(3)$ \\
\hline Ga9-S4 & $2.357(3)$ & K1-C11 & $2.894(4)$ & K3-Cl1 & $2.907(4)$ \\
\hline K2-C11 & $2.930(4)$ & K4-C11 & $2.939(4)$ & & \\
\hline \multicolumn{6}{|c|}{2} \\
\hline bond & distance & bond & distance & bond & distance \\
\hline $\mathrm{Cd} 1-\mathrm{Se} 2$ & $2.601(2)$ & Ga1-Se13 & $2.377(3)$ & Ga2-Se8 & $2.343(3)$ \\
\hline Cd1-Se6 & $2.609(2)$ & Ga1-Se1 & $2.382(2)$ & Ga2-Se6 & $2.446(3)$ \\
\hline Cd1-Se12 & $2.614(3)$ & Ga1-Se10 & $2.417(3)$ & $\mathrm{Ga} 2-\mathrm{Se} 4$ & $2.464(3)$ \\
\hline Cd1-Se10 & $2.615(2)$ & Ga1-Se3 & $2.465(2)$ & Ga2-Se14 & $2.377(3)$ \\
\hline Ga3-Se8 & $2.347(3)$ & Ga4-Se5 & $2.367(3)$ & Ga5-Se11 & $2.351(3)$ \\
\hline Ga3-Se15 & $2.367(3)$ & Ga4-Se6 & $2.390(3)$ & Ga5-Se2 & $2.411(2)$ \\
\hline Ga3-Se10 & $2.430(3)$ & Ga4-Se9 & $2.439(3)$ & Ga5-Se9 & $2.440(3)$ \\
\hline
\end{tabular}




\begin{tabular}{llllll} 
Ga3-Se9 & $2.456(3)$ & Ga4-Se7 & $2.451(2)$ & Ga5-Se3 & $2.453(3)$ \\
Ga6-Se15 & $2.356(3)$ & Ga7-Se16 & $2.364(3)$ & Ga8-Se16 & $2.369(3)$ \\
Ga6-Se1 & $2.370(3)$ & Ga7-Se13 & $2.371(2)$ & Ga8-Se14 & $2.369(3)$ \\
Ga6-Se4 & $2.459(2)$ & Ga7-Se2 & $2.423(3)$ & Ga8-Se12 & $2.416(3)$ \\
Ga6-Se7 & $2.475(3)$ & Ga7-Se7 & $2.494(3)$ & Ga8-Se3 & $2.490(2)$ \\
Ga9-Se11 & $2.362(3)$ & Ga9-Se5 & $2.387(3)$ & Ga9-Se12 & $2.431(3)$ \\
Ga9-Se4 & $2.479(2)$ & K1-Cl1 & $2.907(8)$ & K2-Cl1 & $2.956(7)$ \\
K3-C11 & $2.936(7)$ & K4-C11 & $2.947(7)$ & & \\
\hline
\end{tabular}

Table S3. The laser-induced damage thresholds of 1, 2, and AGS.

\begin{tabular}{lllll}
\hline Compounds & $\begin{array}{l}\text { Damage energy } \\
(\mathrm{mJ})\end{array}$ & $\begin{array}{l}\text { Spot area } \\
\left(\mathrm{cm}^{2}\right)\end{array}$ & $\begin{array}{l}\tau_{\mathrm{p}} \\
(\mathrm{ns})\end{array}$ & $\begin{array}{l}\text { Damage threshold } \\
{\left[\mathrm{MW} \cdot \mathrm{cm}^{-2}\right]}\end{array}$ \\
\hline $\mathbf{1}$ & 9.56 & 0.015 & 10 & 63.7 \\
$\mathbf{2}$ & 3.241 & 0.015 & 10 & 21.6 \\
AGS & 0.423 & 0.015 & 10 & 2.82 \\
\hline
\end{tabular}

Table S4. Partial SHG contributions of atom types in unit cells of $\mathbf{1}$ and $\mathbf{2}$ averaged on all nonzero independent SHG coefficient tensors at the wavelength of $1910 \mathrm{~nm}$.

\begin{tabular}{|c|c|c|c|c|c|}
\hline \multicolumn{6}{|c|}{1} \\
\hline Atom & $\mathrm{S}$ & $\mathrm{Ga}$ & $\mathrm{Cd}$ & $\mathrm{Cl}$ & $\mathrm{K}$ \\
\hline SHG (\%) & 63.70 & 26.36 & 2.90 & 2.89 & 4.15 \\
\hline \multicolumn{6}{|c|}{2} \\
\hline Atom & $\mathrm{Se}$ & $\mathrm{Ga}$ & $\mathrm{Cd}$ & $\mathrm{Cl}$ & $\mathrm{K}$ \\
\hline SHG (\%) & 71.86 & 22.14 & 1.57 & 2.15 & 2.28 \\
\hline
\end{tabular}

Table S5. Distortion indicators, $\Delta H$ and $\Delta R$, of asymmetric $\mathrm{GaQ}_{4}$ tetrahedra in $\mathbf{1}, \mathbf{2}$ and reference compounds.

\begin{tabular}{lllllll}
\hline Compound & $\mathrm{GaQ}_{4}$ & $\Delta H$ & $\Delta R$ & $\mathrm{GaQ}_{4}$ & $\Delta H$ & $\Delta R$ \\
\hline \multicolumn{7}{c}{$\mathrm{GaS}_{4}$} \\
\hline $\mathrm{Ga}(1) \mathrm{S}_{4}$ & 0.0076 & 0.0020 & $\mathrm{Ga}(2) \mathrm{S}_{4}$ & 0.0128 & 0.0033 \\
\hline
\end{tabular}




\begin{tabular}{|c|c|c|c|c|c|c|}
\hline & $\mathrm{Ga}(3) \mathrm{S}_{4}$ & 0.0112 & 0.0029 & $\mathrm{Ga}(4) \mathrm{S}_{4}$ & 0.0059 & 0.0015 \\
\hline \multirow[t]{3}{*}{1} & $\mathrm{Ga}(5) \mathrm{S}_{4}$ & 0.0085 & 0.0021 & $\mathrm{Ga}(6) \mathrm{S}_{4}$ & 0.0153 & 0.0040 \\
\hline & $\mathrm{Ga}(7) \mathrm{S}_{4}$ & 0.0134 & 0.0036 & $\mathrm{Ga}(8) \mathrm{S}_{4}$ & 0.0134 & 0.0036 \\
\hline & $\mathrm{Ga}(9) \mathrm{S}_{4}$ & 0.0080 & 0.0021 & & & \\
\hline \multirow[t]{2}{*}{$\mathrm{Li}\left[\mathrm{LiCs}_{2} \mathrm{Cl}\right]\left[\mathrm{Ga}_{3} \mathrm{~S}_{6}\right]^{5}$} & $\mathrm{Ga}(1) \mathrm{S}_{4}$ & 0.0012 & 0.0003 & $\mathrm{Ga}(2) \mathrm{S}_{4}$ & 0.0003 & 0.0001 \\
\hline & $\mathrm{Ga}(3) \mathrm{S}_{4}$ & 0.0003 & 0.0001 & & & \\
\hline \multirow[t]{2}{*}[\mathrm{RbBa}_{2}\mathrm{Cl}]{$\left[\mathrm{Ga}_{4} \mathrm{~S}_{8}\right]^{6}$} & $\mathrm{Ga}(1) \mathrm{S}_{4}$ & 0.0072 & 0.0019 & $\mathrm{Ga}(2) \mathrm{S}_{4}$ & 0.0051 & 0.0013 \\
\hline & $\mathrm{Ga}(3) \mathrm{S}_{4}$ & 0.0024 & 0.0006 & & & \\
\hline$\left[\mathrm{CsBa}_{2} \mathrm{Cl}\right]\left[\mathrm{Ga}_{4} \mathrm{~S}_{8}\right]^{\text {Error }}$ & $\mathrm{Ga}(1) \mathrm{S}_{4}$ & 0.0081 & 0.0022 & $\mathrm{Ga}(2) \mathrm{S}_{4}$ & 0.0061 & 0.0016 \\
\hline ! Bookmark not defined. & $\mathrm{Ga}(3) \mathrm{S}_{4}$ & 0.0015 & 0.0004 & & & \\
\hline$\left[\mathrm{KBa}_{3} \mathrm{Cl}_{2}\right]\left[\mathrm{Ga}_{5} \mathrm{~S}_{10}\right]^{7}$ & $\mathrm{Ga}(1) \mathrm{S}_{4}$ & 0.0000 & 0.0000 & $\mathrm{Ga}(2) \mathrm{S}_{4}$ & 0.0004 & 0.0001 \\
\hline$\left[\mathrm{RbBa}_{3} \mathrm{Cl}_{2}\right]\left[\mathrm{Ga}_{5} \mathrm{~S}_{10}\right]^{\mathrm{Er}}$ & $\mathrm{Ga}(1) \mathrm{S}_{4}$ & 0.0000 & 0.0000 & $\mathrm{Ga}(2) \mathrm{S}_{4}$ & 0.0003 & 0.0001 \\
\hline \multicolumn{7}{|l|}{ ror! Bookmark not defined. } \\
\hline$\left[\mathrm{CsBa}_{3} \mathrm{Cl}_{2}\right]\left[\mathrm{Ga}_{5} \mathrm{~S}_{10}\right]^{\mathrm{Err}}$ & $\mathrm{Ga}(1) \mathrm{S}_{4}$ & 0.0000 & 0.0000 & $\mathrm{Ga}(2) \mathrm{S}_{4}$ & 0.0003 & 0.0001 \\
\hline \multicolumn{7}{|c|}{$\mathrm{GaSe}_{4}$} \\
\hline \multirow{5}{*}{2} & $\mathrm{Ga}(1) \mathrm{Se}_{4}$ & 0.0063 & 0.0016 & $\mathrm{Ga}(2) \mathrm{Se}_{4}$ & 0.0129 & 0.0033 \\
\hline & $\mathrm{Ga}(3) \mathrm{Se}_{4}$ & 0.0103 & 0.0023 & $\mathrm{Ga}(4) \mathrm{Se}_{4}$ & 0.0063 & 0.0016 \\
\hline & $\mathrm{Ga}(5) \mathrm{Se}_{4}$ & 0.0086 & 0.0022 & $\mathrm{Ga}(6) \mathrm{Se}_{4}$ & 0.0145 & 0.0037 \\
\hline & $\mathrm{Ga}(7) \mathrm{Se}_{4}$ & 0.0136 & 0.0036 & $\mathrm{Ga}(8) \mathrm{Se}_{4}$ & 0.0120 & 0.0032 \\
\hline & $\mathrm{Ga}(9) \mathrm{Se}_{4}$ & 0.0101 & 0.0026 & & & \\
\hline $\mathrm{Ba}_{3} \mathrm{Cl}_{2} \mathrm{KGa}_{5} \mathrm{Se}_{10}^{8}$ & $\mathrm{Ga}(1) \mathrm{Se}_{4}$ & 0.0004 & 0.0001 & $\mathrm{Ga}(2) \mathrm{Se}_{4}$ & 0.0000 & 0.0000 \\
\hline $\mathrm{Ba}_{3} \mathrm{Cl}_{2} \mathrm{RbGa}_{5} \mathrm{Se}_{10}$ Error! & $\mathrm{Ga}(1) \mathrm{Se}_{4}$ & 0.0004 & 0.0001 & $\mathrm{Ga}(2) \mathrm{Se}_{4}$ & 0.0000 & 0.0000 \\
\hline \multicolumn{7}{|l|}{ Bookmark not defined. } \\
\hline $\mathrm{Ba}_{3} \mathrm{Cl}_{2} \mathrm{CsGa}_{5} \mathrm{Se}_{10}$ Error! & $\mathrm{Ga}(1) \mathrm{Se}_{4}$ & 0.0003 & 0.0001 & $\mathrm{Ga}(2) \mathrm{Se}_{4}$ & 0.0000 & 0.0000 \\
\hline Bookmark not defined. & & & & & & \\
\hline
\end{tabular}

Table S6. Comparison of the SHG responses of the salt-inclusion chalcogenides reported in the past decade. 


\begin{tabular}{|c|c|c|c|}
\hline Compounds & Space group & SHG $(× \mathrm{AGS})$ & $\mathrm{PM} / \mathrm{NPM}$ \\
\hline$\left[\mathrm{Li}_{2} \mathrm{Cs}_{2} \mathrm{Cl}\right]\left[\mathrm{Ga}_{3} \mathrm{~S}_{6}\right]^{5}$ & $\operatorname{Pna2}_{1}$ & 0.7 & PM \\
\hline$\left[\mathrm{K}_{3} \mathrm{Cl}\right]\left[\mathrm{PGa}_{3} \mathrm{~S}_{8}\right]^{9}$ & $P m n 2_{1}$ & 1.0 & $\mathrm{PM}$ \\
\hline$\left[\mathrm{Rb}_{3} \mathrm{Cl}\right]\left[\mathrm{PGa}_{3} \mathrm{~S}_{8}\right]^{9}$ & Pmn21 & 1.1 & $\mathrm{PM}$ \\
\hline$\left[\mathrm{K}_{3} \mathrm{Br}\right]\left[\mathrm{PGa}_{3} \mathrm{~S}_{8}\right]^{9}$ & $P m$ & 1.2 & $\mathrm{PM}$ \\
\hline$\left[\mathrm{Rb}_{3} \mathrm{Br}\right]\left[\mathrm{PGa}_{3} \mathrm{~S}_{8}\right]^{9}$ & $P m$ & 2.0 & PM \\
\hline$\left[\mathrm{KBa}_{3} \mathrm{Cl}_{2}\right]\left[\mathrm{Ga}_{5} \mathrm{~S}_{10}\right]^{7}$ & $I \overline{4}$ & 0.9 & $\mathrm{PM}$ \\
\hline$\left[\mathrm{RbBa}_{3} \mathrm{Cl}_{2}\right]\left[\mathrm{Ga}_{5} \mathrm{~S}_{10}\right]^{7}$ & $I \overline{4}$ & 0.93 & $\mathrm{PM}$ \\
\hline$\left[\mathrm{CsBa}_{3} \mathrm{Cl}_{2}\right]\left[\mathrm{Ga}_{5} \mathrm{~S}_{10}\right]^{7}$ & $I \overline{4}$ & 0.95 & $\mathrm{PM}$ \\
\hline$\left[\mathrm{RbBa}_{2} \mathrm{Cl}\right]\left[\mathrm{Ga}_{4} \mathrm{~S}_{8}\right]^{6}$ & $I \overline{4}$ & 1.0 & $\mathrm{PM}$ \\
\hline$\left[\mathrm{CsBa}_{2} \mathrm{Cl}\right]\left[\mathrm{Ga}_{4} \mathrm{~S}_{8}\right]^{6}$ & $I \overline{4}$ & 0.9 & $\mathrm{PM}$ \\
\hline$\left[\mathrm{Ba}_{4} \mathrm{Cl}_{2}\right]\left[\mathrm{ZnGa}_{4} \mathrm{~S}_{10}\right]^{10}$ & $I \overline{4}$ & 1.1 & $\mathrm{PM}$ \\
\hline$\left[\mathrm{KBa}_{3} \mathrm{Cl}_{2}\right]\left[\mathrm{Ga}_{5} \mathrm{Se}_{10}\right]^{8}$ & $I \overline{4}$ & 10 & NPM \\
\hline$\left[\mathrm{RbBa}_{3} \mathrm{Cl}_{2}\right]\left[\mathrm{Ga}_{5} \mathrm{Se}_{10}\right]^{8}$ & $I \overline{4}$ & 20 & NPM \\
\hline$\left[\mathrm{CsBa}_{3} \mathrm{Cl}_{2}\right]\left[\mathrm{Ga}_{5} \mathrm{Se}_{10}\right]^{8}$ & $I \overline{4}$ & 100 & NPM \\
\hline$\left[\mathrm{Ba}_{4} \mathrm{Cl}_{2}\right]\left[\mathrm{ZnGa}_{4} \mathrm{Se}_{10}\right]^{11}$ & $I \overline{4}$ & 59 & NPM \\
\hline$\left[\mathrm{Ba}_{4} \mathrm{Cl}_{2}\right]\left[\mathrm{MnGa}_{4} \mathrm{Se}_{10}\right]^{11}$ & $I \overline{4}$ & 30 & NPM \\
\hline$\left[\mathrm{Ba}_{4} \mathrm{Cl}_{2}\right]\left[\mathrm{CdGa}_{4} \mathrm{Se}_{10}\right]^{11}$ & $I \overline{4}$ & 52 & NPM \\
\hline$\left[\mathrm{Ba}_{4} \mathrm{Cl}_{2}\right]\left[\mathrm{Cu}_{0.5} \mathrm{Ga}_{4.5} \mathrm{Se}_{10}\right]^{11}$ & $I \overline{4}$ & 39 & NPM \\
\hline$\left[\mathrm{CsBa}_{3} \mathrm{Cl}_{2}\right]\left[\mathrm{In}_{0.5} \mathrm{Ga} 4.5 \mathrm{Se}_{10}\right]^{12}$ & $I \overline{4}$ & 64 & NPM \\
\hline$\left[\mathrm{CsBa}_{3} \mathrm{Cl}_{2}\right]\left[\mathrm{InGa}_{4} \mathrm{Se}_{10}\right]^{12}$ & $I \overline{4}$ & 70 & NPM \\
\hline$\left[\mathrm{K}_{3} \mathrm{I}\right]\left[\mathrm{SmB}_{12}\left(\mathrm{GaS}_{4}\right)_{3}\right]^{13}$ & $P 6_{3} 22$ & $0.3 \times \mathrm{KDP}$ & NA \\
\hline$\left[\mathrm{K}_{3} \mathrm{I}\right]\left[\operatorname{InB}_{12}\left(\mathrm{InSe}_{4}\right)_{3}\right]^{14}$ & $P 6_{3} 22$ & $1 / 14 \times \mathrm{KTP}$ & NA \\
\hline$\left[\mathrm{Ba}_{4} \mathrm{Cl}_{2}\right]\left[\mathrm{Ge}_{3} \mathrm{~S}_{9}\right]^{15}$ & $P 6_{3}$ & 2.4 & NPM \\
\hline$\left[\mathrm{Ba}_{4} \mathrm{Cl}_{2}\right]\left[\mathrm{Ge}_{3} \mathrm{Se}_{9}\right]^{15}$ & $P 6_{3}$ & 1.0 & NPM \\
\hline$\left[\mathrm{Ba}_{4} \mathrm{Cl}_{2}\right]\left[\mathrm{Si}_{3} \mathrm{Se}_{9}\right]^{15}$ & $P 6_{3}$ & 1.0 & NPM \\
\hline$\left[\mathrm{NaBa}_{4} \mathrm{Cl}\right]\left[\mathrm{Ge}_{3} \mathrm{~S}_{10}\right]^{16}$ & $P 6_{3}$ & 0.33 & NA \\
\hline$\left[\mathrm{Ba}_{4} \mathrm{Br}_{2}\right]\left[\mathrm{Si}_{3} \mathrm{Se}_{9}\right]^{17}$ & $P 6_{3}$ & 3.2 & NPM \\
\hline$\left[\mathrm{Ba}_{4} \mathrm{Br}_{2}\right]\left[\mathrm{Ge}_{3} \mathrm{Se}_{9}\right]^{17}$ & $\mathrm{PG}_{3}$ & 3.5 & NPM \\
\hline
\end{tabular}




\begin{tabular}{|l|l|l|l|}
\hline$\left[\mathrm{K}_{4} \mathrm{Cl}\right]\left[\mathrm{CdGa}_{9} \mathrm{~S}_{16}\right]^{\text {this work }}$ & $P 1$ & 0.9 & PM \\
\hline$\left[\mathrm{K}_{4} \mathrm{Cl}\right]\left[\mathrm{CdGa}_{9} \mathrm{Se}_{16}\right]^{\text {this work }}$ & $P 1$ & 2.4 & PM \\
\hline
\end{tabular}

$\mathrm{NA}=$ Not Available.

\section{References}

1. Lalik, E. Shannon information as a measure of distortion in coordination polyhedra. J. Appl. Cryst. 2005, 38, 152.

2. I. D. Brown. The Chemical Bond in Inorganic Chemistry: The Bond Valence Model. Oxford University Press, 2002.

3. https://www.iucr.org/resources/data/datasets/bond valence-parameters.

4. Brown, I. D. On measuring the size of distortions in coordination polyhedra. Acta Cryst. 2006, B62, 692.

5. Liu, B.-W.; Jiang, X.-M.; Li, B.-X.; Zeng, H.-Y.; Guo, G.-C., Li[LiCs $\left.{ }_{2} \mathrm{Cl}_{[}\right]\left[\mathrm{Ga}_{3} \mathrm{~S}_{6}\right]$ : A nanoporous framework of GaS4 tetrahedra with excellent nonlinear optical performance. Angew. Chem. Int. Ed. 2020, 59, 4856-4859.

6. Liu, B.-W.; Jiang, X.-M.; Zeng, H.-Y.; Guo, G.-C. $\left[\mathrm{ABa}_{2} \mathrm{Cl}\right]\left[\mathrm{Ga}_{4} \mathrm{~S}_{8}\right](\mathrm{A}=\mathrm{Rb}, \mathrm{Cs})$ : Widespectrum nonlinear optical materials obtained by polycation-substitution-induced nonlinear optical (NLO) functional motif ordering. J. Am. Chem. Soc. 2020, 142, 10641-10645.

7. Liu, B.-W.; Zeng, H.-Y.; Jiang, X.-M.; Guo, G.-C. Phase matching achieved by bandgap widening in infrared nonlinear optical materials $\left[\mathrm{ABa}_{3} \mathrm{Cl}_{2}\right]\left[\mathrm{Ga}_{5} \mathrm{~S}_{10}\right](\mathrm{A}=\mathrm{K}, \mathrm{Rb}$, and $\mathrm{Cs})$. $C C S$ Chem. 2020, 2, 964-973.

8. Yu, P.; Zhou, L. J.; Chen, L. Noncentrosymmetric Inorganic Open-framework chalcohalides with strong middle IR SHG and red emission $\mathrm{ABa}_{3} \mathrm{Ga}_{5} \mathrm{Se}_{10} \mathrm{Cl}_{2}(\mathrm{~A}=\mathrm{Cs}, \mathrm{Rb}, \mathrm{K})$. J. Am. Chem. 
Soc. 2012, 134, 2227-2235.

9 Liu, B.-W.; Zeng, H.-Y.; Jiang, X.-M.; Wang, G.-E.; Li, S.-F.; Xu, L.; Guo, G.-C.

$\left[\mathrm{A}_{3} \mathrm{X}\right]\left[\mathrm{Ga}_{3} \mathrm{PS}_{8}\right](\mathrm{A}=\mathrm{K}, \mathrm{Rb} ; \mathrm{X}=\mathrm{Cl}, \mathrm{Br})$ : Promising IR nonlinear optical materials exhibiting concurrently strong second-harmonic generation and high laser induced damage thresholds.

Chem. Sci. 2016, 7, 6273-6277.

10 Chen, H.; Li, Y. Y.; Li, B. X.; Liu, P. F.; Lin, H.; Zhu, Q. L.; Zhu, Q. L. Salt-inclusion chalcogenide $\left[\mathrm{Ba}_{4} \mathrm{Cl}_{2}\right]\left[\mathrm{ZnGa}_{4} \mathrm{~S}_{10}\right]$ : rational design of an IR nonlinear optical material with superior comprehensive performance derived from $\mathrm{AgGaS}_{2}$. Chem. Mater. 2020, 32, 8012-8019.

11 Li, Y. Y.; Liu, P. F.; Hu, L.; Chen, L.; Lin, H.; Zhou, L. J.; Wu, L. M. Strong IR NLO Material $\mathrm{Ba}_{4} \mathrm{MGa}_{4} \mathrm{Se}_{10} \mathrm{Cl}_{2}$ : Highly Improved Laser Damage Threshold via Dual Ion Substitution Synergy. Adv. Opt. Mater. 2015, 3, 957-966.

12 Li, Y. Y.; Liu, P. F.; Lin, H.; Wang, M. T.; Chen, L. The effect of indium substitution on the structure and NLO properties of $\mathrm{Ba}_{6} \mathrm{Cs}_{2} \mathrm{Ga}_{10} \mathrm{Se}_{20} \mathrm{Cl}_{4}$. Inorg. Chem. Front. 2016, 3, 952958.

13 Sun, Z.-D.; Chi, Y.; Xue, H.-G.; Guo, S.-P. Synthesis, crystal structure and second-order nonlinear optical property of a novel pentanary selenide $\left(\mathrm{K}_{3} \mathrm{I}\right)\left[\operatorname{InB}_{12}\left(\operatorname{InSe}_{4}\right)_{3}\right]$. Inorg. Chem. Front. 2017, 4, 1841-1847. 
14 Guo, S.-P.; Chi, Y.; Liu, B.-W.; Guo, G.-C. Synthesis, crystal structure and second-order nonlinear optical property of a novel pentanary selenide $\left(\mathrm{K}_{3} \mathrm{I}\right)\left[\operatorname{InB}_{12}\left(\operatorname{InSe}_{4}\right)_{3}\right]$. Dalton Trans. 2016, 45, 10459-10465.

15 Liu, P. F.; Li, Y. Y.; Zheng, Y. J.; Yu, J. S.; Duan, R. H.; Chen, H.; Lin, H.; Chen, L. Wu, L. M.; Tailored synthesis of nonlinear optical quaternary chalcohalides: $\mathrm{Ba}_{4} \mathrm{Ge}_{3} \mathrm{~S}_{9} \mathrm{Cl}_{2}$, $\mathrm{Ba}_{4} \mathrm{Si}_{3} \mathrm{Se}_{9} \mathrm{Cl}_{2}$ and $\mathrm{Ba}_{4} \mathrm{Ge}_{3} \mathrm{Se}_{9} \mathrm{Cl}_{2}$. Dalton Trans. 2017, 46, 2715-2721.

16 Feng, K.; Kang, L.; Lin, Z. S.; Yao, J. Y.; Wu, Y. C. Noncentrosymmetric chalcohalide $\mathrm{NaBa}_{4} \mathrm{Ge}_{3} \mathrm{~S}_{10} \mathrm{Cl}$ with large band gap and IR NLO response. J. Mater. Chem. C, 2014, 2, 45904596.

17 Xing, W.; Wang, N.; Iyer, A. K.; Yin, W.; Lin, Z.; Yao, J.; Kang, B. Mar, A. Evaluation of nonlinear optical properties of quaternary chalcogenide halides $\mathrm{Ba}_{4} \mathrm{Si}_{3} \mathrm{Se}_{9} \mathrm{Br}_{2}$ and $\mathrm{Ba}_{4} \mathrm{Ge}_{3} \mathrm{Se}_{9} \mathrm{Br}_{2}$. J. Alloys Compd. 2020, 846, 156398. 